\title{
Direct Intraesophageal Growth from Metastatic Mediastinal Lymphadenopathy in Thymic Carcinoma
}

\author{
Hiroki Kanazawa ${ }^{1}$, Nobukazu Sasaki ${ }^{1}$, Takashi Kobayashi ${ }^{2}$, Toshirou Fukushima ${ }^{2}$, \\ Shintaro Kanda ${ }^{2}$, Tomonobu Koizumi ${ }^{2}$ and Mai Iwaya ${ }^{3}$
}

\begin{abstract}
:
We herein report a case of thymic carcinoma that initially exhibited dysphagia and an intraesophageal mass lesion. A 68-year-old man was admitted to our hospital because of dysphagia. An endoscopic examination revealed a mass on the middle esophagus. Chest computed tomography (CT) showed a huge anterior mediastinal mass and subcarinal lymph node swelling, directly invading into the esophageal lumen. An immunohistological examination of the esophageal and anterior mediastinal masses revealed squamous cell carcinoma originating from the thymus. This is the first report of a thymic carcinoma spreading into the esophageal lumen and forming a mass lesion.
\end{abstract}

Key words: mediastinal tumor, subcarinal lymph node, ADOC chemotherapy, esophagus

(Intern Med 60: 923-926, 2021)

(DOI: 10.2169/internalmedicine.5501-20)

\section{Introduction}

Thymic carcinoma is a thymic epithelial neoplasm with cytological malignant features and a clinical course that tends to be much more aggressive than that of thymoma $(1,2)$. Thymic carcinoma, located in the anterosuperior mediastinum, frequently spreads to the pleural space, regional lymph nodes, liver, and lung (1-6). The initial clinical presentations in patients with thymic carcinoma include chest pain, cough, and superior vena cava syndrome $(5,6)$. In addition, due to the paucity of cases, there is little information about the efficacy of diagnostic and therapeutic approaches in clinical practice.

We encountered a case of thymic carcinoma initially showing dysphagia. Endoscopic and radiological findings revealed an intraesophageal mass directly expanding from metastatic subcarinal lymphadenopathy due to thymic carcinoma in the anterior mediastinum. We herein report the clinical course and treatment outcome.

\section{Case Report}

A 68-year-old man presented with dysphagia that progressed gradually over 2 months. Body weight loss $(2 \mathrm{~kg} /$ month) was observed. Physical and laboratory examinations were unremarkable. An endoscopic examination revealed a mass on the middle intrathoracic esophagus (Fig. 1A). He was referred to our hospital for a further examination.

Chest computed tomography (CT) demonstrated a huge anterior mediastinal mass and swelling of the subcarinal lymph node (Fig. 2A). The subcarinal mass extended directly into the esophageal lumen (Fig. 2B). A CT-guided percutaneous biopsy of the anterior mediastinal mass and endoscopic biopsy of the esophageal mass were performed. The histopathological findings in both specimens revealed squamous cell carcinoma (Fig. 3A, D).

On an immunohistological analysis, the tumor cells in the anterior mediastinal mass were positive for CD5 (Fig. 3B), while those in the esophageal mass were weak (Fig. 3E), but CD63 was positive in both specimens (Fig. 3C, F). Based on the radiological and histopathological findings, the esophageal mass seemed to be due to the direct invasion of

${ }^{1}$ Second Department of Internal Medicine, Shinshu University School of Medicine, Japan, ${ }^{2}$ Department of Comprehensive Cancer Therapy, Shinshu University School of Medicine, Japan and ${ }^{3}$ Department of Centarl Laboratory, Shinshu University School of Medicine, Japan Received: June 2, 2020; Accepted: September 3, 2020; Advance Publication by J-STAGE: October 21, 2020 Correspondence to Dr. Tomonobu Koizumi, tomonobu@shinshu-u.ac.jp 


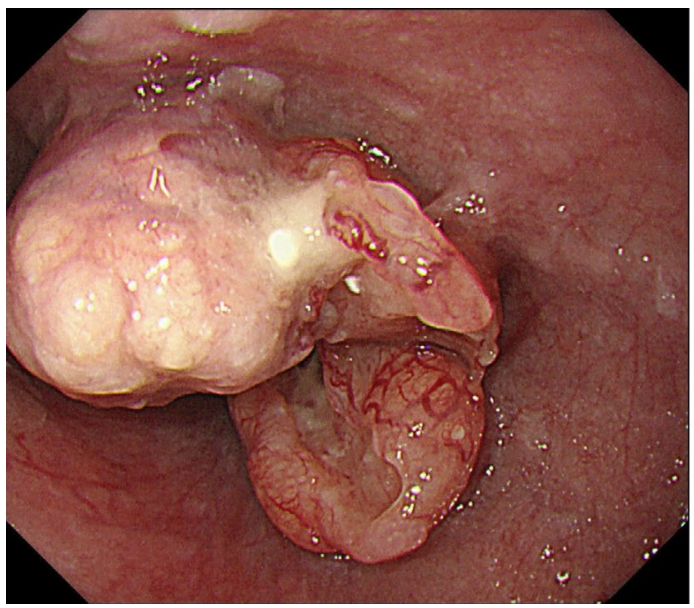

A

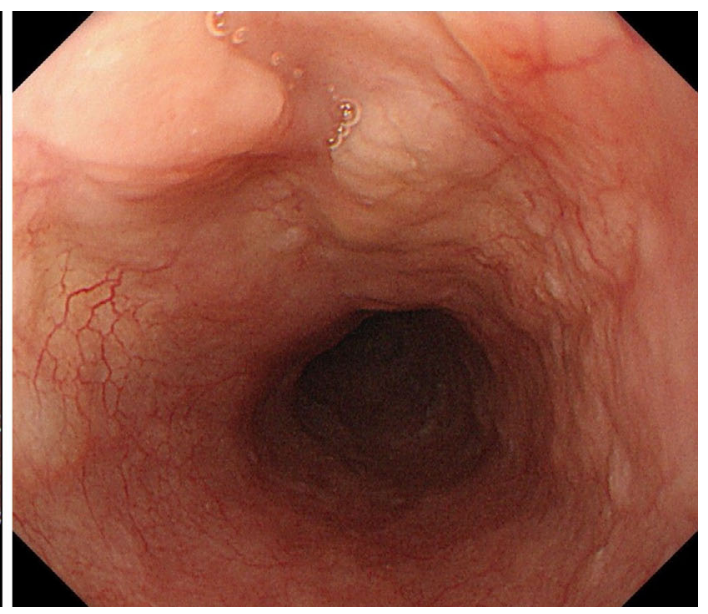

B

Figure 1. Endoscopic findings on admission (A) revealed an elevated, irregular mass with ulcerative lesions in esophagus. The mass lesion disappeared after four cycles of ADOC chemotherapy (B).

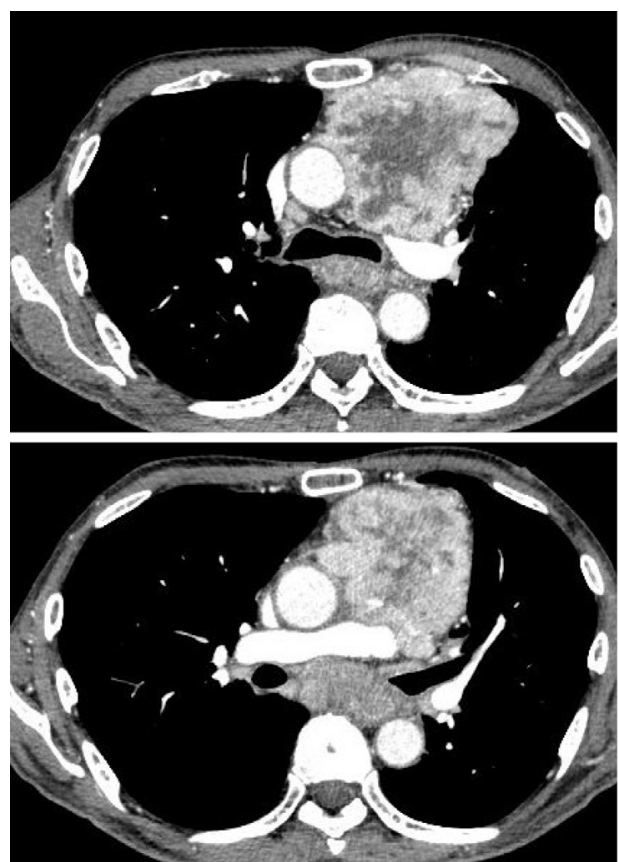

A

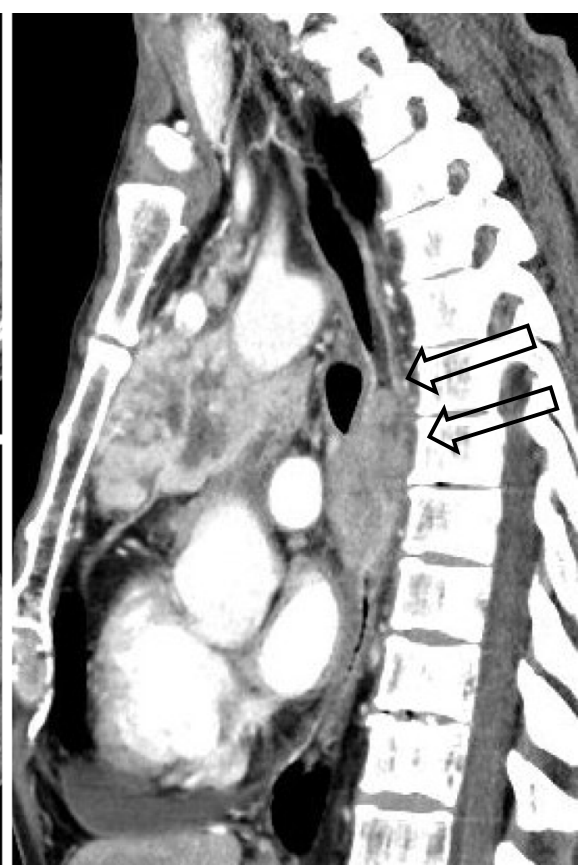

B

Figure 2. Chest computed tomography (CT) showed a huge anterior mediastinal mass and subcarinal lymphadenopathy (A). Coronal views on chest CT showed that the subcarinal lymph node had invaded directly into the esophageal lumen (B; arrows).

metastatic cancer cells via the mediastinal lymph node from the thymic carcinoma located in the anterior mediastinum. There were no other distant metastatic lesions.

Since thoracic radiotherapy for the whole tumor was considered difficult due to the large field, the patient received chemotherapy with a combination of cisplatin $\left(50 \mathrm{mg} / \mathrm{m}^{2}\right)$ and doxorubicin $\left(40 \mathrm{mg} / \mathrm{m}^{2}\right)$ on day 1 , vincristine $(0.6 \mathrm{mg}$ / $\mathrm{m}^{2}$ ) on day 3 , and cyclophosphamide $\left(700 \mathrm{mg} / \mathrm{m}^{2}\right)$ on day 4 (ADOC chemotherapy). Four cycles of ADOC chemotherapy resulted in a marked reduction in both tumors, especially in the lesion in the mediastinal lymph node (Fig. 4).
The endoscopic findings also improved, and the intraesophageal mass disappeared almost completely (Fig. 1B). The symptom of dysphagia also improved. Thoracic radiotherapy $(2$ Gy $\times 30$ fractions, total $60 \mathrm{~Gy})$ was subsequently applied, and the patient has remained well for approximately 6 months since the diagnosis.

\section{Discussion}

We encountered a case of thymic carcinoma developing direct invasion into the esophageal lumen from metastatic 

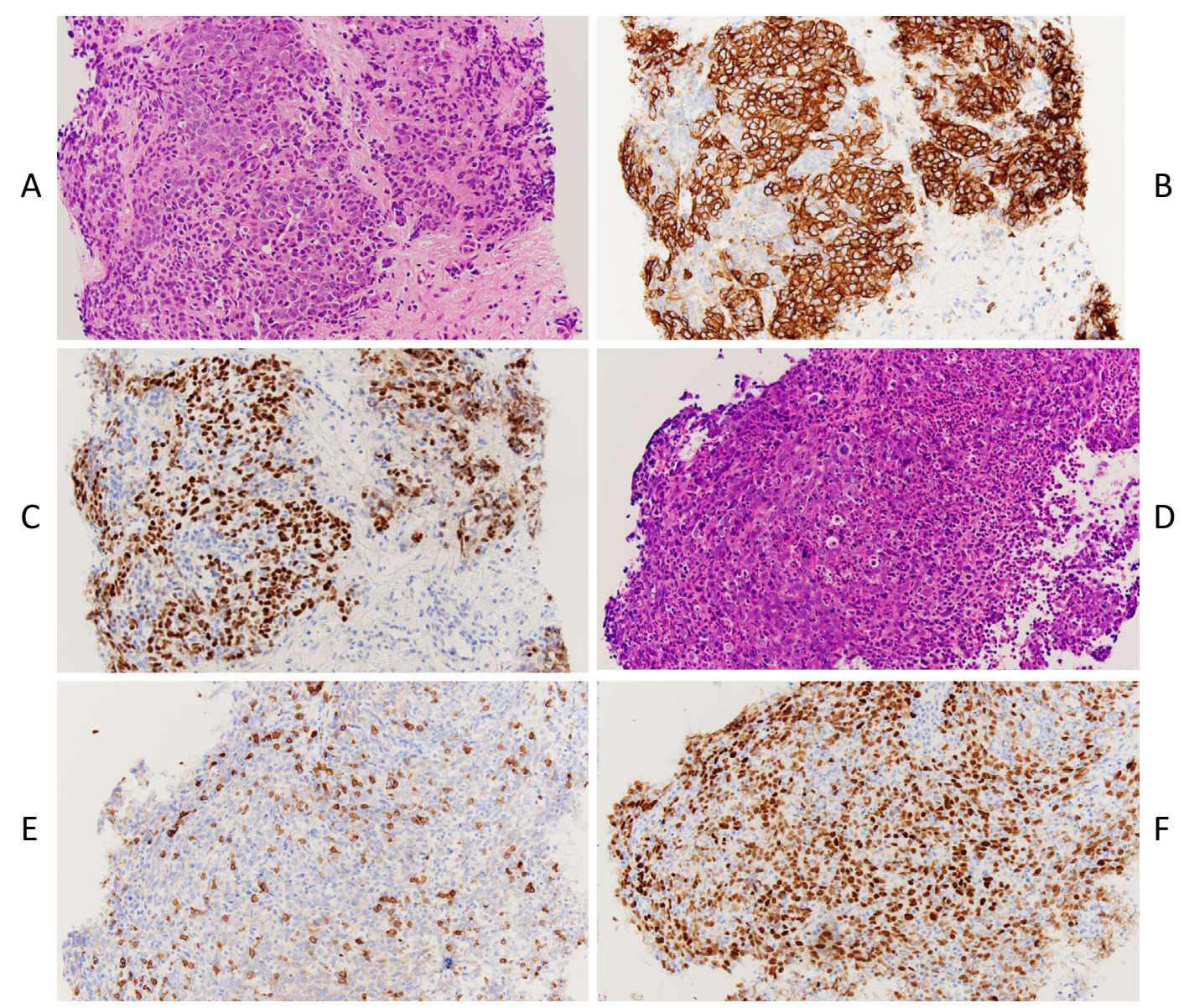

Figure 3. An immunohistological analysis identified the anterior mediastinal mass as squamous cell carcinoma [Hematoxylin and Eosin (H\&E) staining] (A), and the tumor cells were positive for CD5 (B) and CD63 (C). An immunohistological analysis identified the esophageal mass as squamous cell carcinoma (H\&E staining) (D), and the tumor cells were positive for CD 63 (F) but weak for CD5 (E).

mediastinal lymphadenopathy. Intraesophageal growth and/or the occasional development of bronchoesophageal fistula are rare clinical manifestations, but the diagnosis and treatment have proven challenging in our clinical practice.

Among the causative malignant diseases, lung cancer is the most common, and the primary tumor and/or metastatic mediastinal lymph node directly expand into the esophagus wall. In general, the manifestations often present during the late or end stage of the disease (7). With regard to epithelial thymic tumors, several case reports have indicated the direct compression of the esophageal wall by epithelial thymic tumors $(8,9)$. However, direct invasion and formation of a tumor mass in the esophageal lumen are extremely rare in epithelial thymic tumors.

Ozawa et al. (10) reported a case of direct growth into the esophageal lumen from a thymoma (type B3) located in the upper mediastinum. However, to our knowledge, direct invasion and formation of a mass in the esophageal lumen in a patient with thymic carcinoma has not been reported previously.

ADOC chemotherapy is a useful regimen for advanced and metastatic thymic carcinoma $(4,6)$ but not esophageal carcinoma. Four cycles of ADOC chemotherapy were effec- tive for both the anterior mediastinal and intraesophageal masses in our case. In particular, the intraesophageal tumor disappeared almost completely without fistula development. Thoracic radiotherapy was performed in the present case, but caution should be exercised in esophageal lesions, especially with regard to fistula development.

The endoscopic appearance of the esophageal tumor at the initial diagnosis was not distinguishable from primary esophageal carcinoma in the present case, and the histological type was squamous cell carcinoma. Thus, there was a possibility that primary esophageal carcinoma progressed to middle and anterior mediastinal lymph node metastases in the present case. In addition, p63 immunohistological stain, a specific marker for squamous cell carcinoma, was positive in both specimens. However, the anterior mediastinal tumor was positive for CD5, confirming that the tumor was of thymic squamous cell carcinoma origin. CD5 is useful in the differential diagnosis between thymic carcinoma and metastatic squamous cell carcinoma of various primary sites $(11,12)$. Unfortunately, the immunostaining with CD5 in esophageal tumor cells was weak and/or negative in our case. However, we believed that chest CT findings showing expansion directly into the esophagus and the response to 


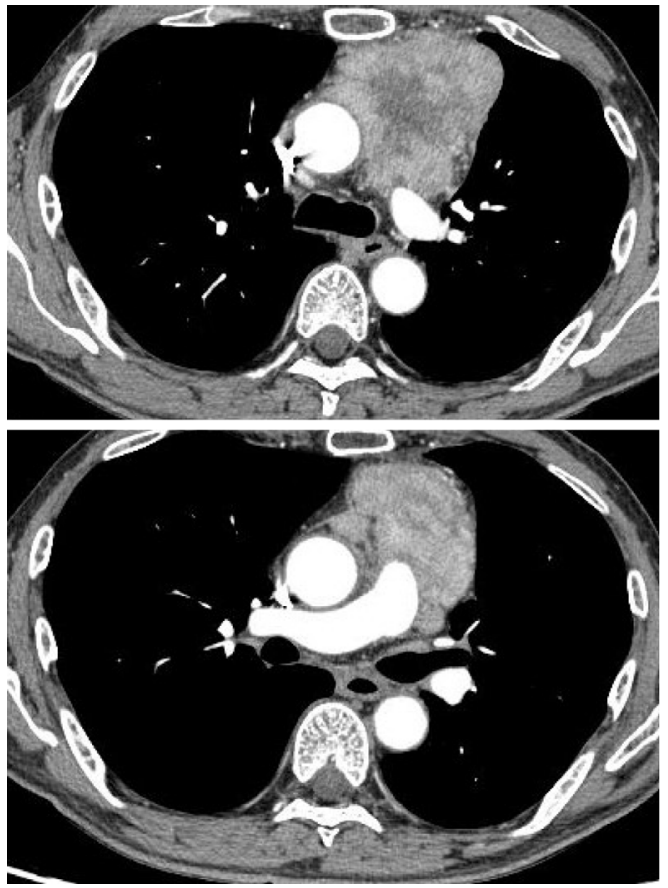

A

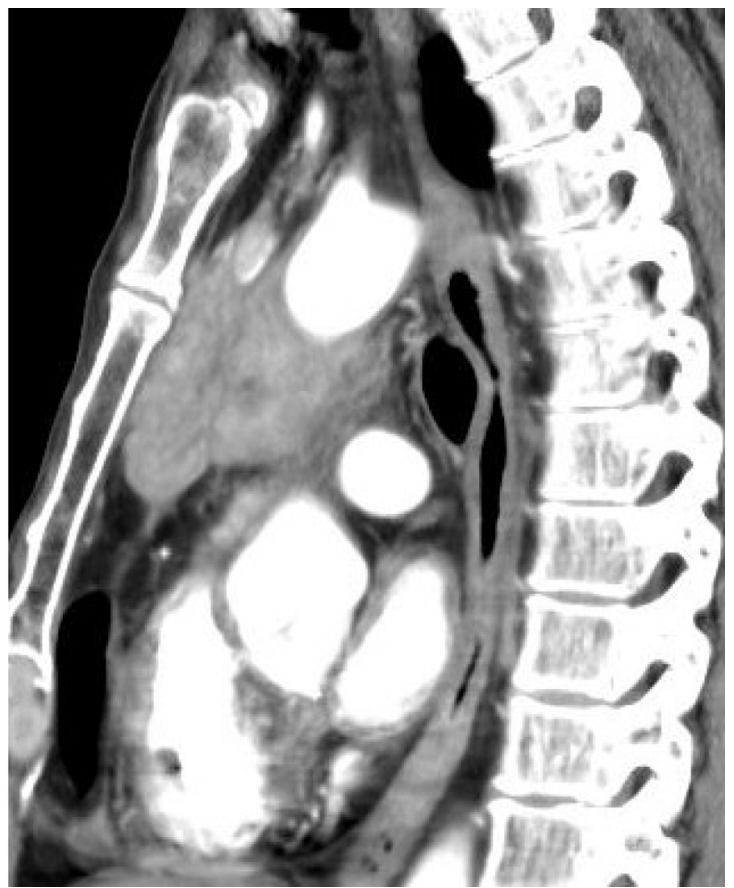

B

Figure 4. Chest computed tomography (CT) after four cycles of ADOC (cisplatin, doxorubicin, vincristine, and cyclophosphamide) chemotherapy showed that the anterior mass was reduced, and subcarinal lymphadenopathy had also disappeared (A). Coronal views on chest CT showed that the tumor invading the esophageal lumen had also disappeared (B).

ADOC chemotherapy supported the esophageal involvement of thymic carcinoma.

In conclusion, we described the first case of intraesophageal growth from thymic carcinoma. The manifestation was unique with regard to the initial presentation of thymic carcinoma. In addition, chemotherapy was useful for treating the disease. The clinical features in the present case may provide new insight into epithelial thymic neoplasms.

The authors state that they have no Conflict of Interest (COI).

\section{References}

1. Hsu CP, Chen CY, Chen CL, Hsu NY, Wang JH, Wang PY. Thymic carcinoma. Ten years' experience in twenty patients. J Thorac Cardiovasc Surg 107: 615-620, 1994.

2. Kondo K, Monden Y. Therapy for thymic epithelial tumors: a clinical study of 1,320 patients from Japan. Ann Thorac Surg 76: 878-885, 2003.

3. Weksler B, Dhupar R, Parikh V, Nason KS, Pennathur A, Ferson $\mathrm{PF}$. Thymic carcinoma: a multivariate analysis of factors predictive of survival in 290 patients. Ann Thorac Surg 95: 299-303, 2013.

4. Ko R, Shukuya T, Okuma Y, et al. Prognostic factors and efficacy of first-line chemotherapy in patients with advanced thymic carcinoma: a retrospective analysis of 286 patients from NEJ023 study. Oncologist 23: 1210-1217, 2018.
5. Yano M, Sasaki H, Yokoyama T, et al. Thymic carcinoma 30 cases at a single institution. J Thoracic Oncol 3: 265-269, 2008.

6. Agatsuma T, Koizumi T, Kanda S, et al. Combination chemotherapy with doxorubicin, vincristine, cyclophosphamide, and platinum compounds for advanced thymic carcinoma. J Thoracic Oncol 12: 2130-2134, 2011.

7. Abugroun A, Ahmed F, Singh N, Nadiri M. Late onset chemo/radiation induced tracheoesophageal fistula in squamous cell cancer of the lung. World J Oncol 8: 171-173, 2017.

8. Oyama K, Onuki T, Mae M, et al. Combined thoracic aortic or upper digestive tract resection for lung cancer and malignant mediastinal tumor. Jpn J Thorac Cardiovasc Surg 48: 9-15, 2000.

9. Ko SF, Tsai YH, Huang HY, et al. Retrotracheal thymoma masquerading as esophageal submucosal tumor. World J Gastroenterol 11: 3165-3166, 2005.

10. Ozawa M, Fukushima T, Noguchi T, Kobayashi T, Sekiguchi N, Koizumi T. Intra-oesophageal invasion of thymoma. Respirol Case Rep 7: e00485, 2019.

11. Hishima T, Fukayama M, Fujisawa M, et al. CD5 expression in thymic carcinoma. Am J Pathol 145: 268-275, 1994.

12. Tateyama H, Eimoto T, Tada T, Hattori H, Murase T, Takino H. Immunoreactivity of a new CD5 antibody with normal epithelium and malignant tumors including thymic carcinoma. Am J Clin Pathol 111: 235-240, 1999 .

The Internal Medicine is an Open Access journal distributed under the Creative Commons Attribution-NonCommercial-NoDerivatives 4.0 International License. To view the details of this license, please visit (https://creativecommons.org/licenses/ by-nc-nd/4.0/).

(C) 2021 The Japanese Society of Internal Medicine Intern Med 60: 923-926, 2021 